\title{
Edge of magnetized electronegative plasma ion source in the presence of collisional adiabatic thermal positive ions
}

\author{
Kiomars Yasserian $^{1}$ (i) $\cdot$ Morteza Aslaninejad $^{2}$
}

Received: 15 April 2019 / Accepted: 2 August 2019 / Published online: 12 August 2019

(c) The Author(s) 2019

\begin{abstract}
This paper develops a theoretical model for formation of multilayers in the magnetized electronegative plasma at the edge of plasma ion source. The impacts of positive ion temperature and collisions are studied and quantified by obtaining the structure of the plasma sheath. By adding the negative ions into the discharge, the solutions of Poisson's equation become oscillatory. A finite temperature for the positive ions and also the collisions results in a change in the behavior of such oscillatory solutions. Here, it is assumed that the collision frequency depends on the positive ions velocity and thermal positive ions flow is adiabatic. The spatial distribution of the species density, electric potential, and positive ion velocity is calculated for different values of positive ion temperature and negative ion concentration for two limiting cases where the collision frequency either is constant or depends linearly on velocity. In addition, the influence of the plasma parameters such as negative ion density and temperature and positive ion temperature is investigated on the space charge and positive ion flux as well as parameter space region. It is also shown that the presence of the negative ion into the plasma ion source influences the extracted positive ion flux and increases the positive ions intensity.
\end{abstract}

keywords Magnetized electronegative plasma - Adiabatic thermal positive ions · Presheath-Sheath · Parameter space region

\section{Introduction}

All of the industrial and laboratory plasmas are confined in a vessel, and many applications of the plasmas come from the interaction of the plasma with the surrounding walls. In addition, the interpretation of results of the active plasma diagnostics such as Langmuir probe immersed in plasma depends on the interaction of the plasma with the solid walls. Therefore, it is essential to understand the plasma-solid interaction features.

In addition, to design an ion source for an accelerator, the properties of the extracted ion have a great influence on the beam characteristics. In general, the ion sources consist of two parts: a plasma generator and ion extraction system. At the extraction region, the plasma is confined to an electrode

Kiomars Yasserian

kiomars.yaserian@kiau.ac.ir

1 Department of Physics, Karaj Branch, Islamic Azad University, Karaj, Iran

2 School of Particles and Accelerators, Institute for Research in Fundamental Sciences (IPM), P.O. Box 19395-5531, Tehran, Iran with a hole in the middle where the plasma sheath is formed. The space charge in the extract region is responsible for divergence of the positive ion. The ion is accelerated through the space charge in which the beam of the ion is diverged. Therefore, it is essential to predict the positive ion flux in the vicinity of the electrodes as a function of plasma parameters.

The plasma properties such as density and energy of the plasma particles depend on several parameters such as the chemical composition of the given plasma. The electronegative gases such as oxygen, chlorine, and fluorocarbons are used extensively in the industry and laboratory for material processing, Langmuir probe diagnostics, and plasma sheath lenses with focusing effects on the charged particle beams. The presence of the negative ions can change the main structure of the plasma sheath transition region where for some ranges of plasma parameters, the multilayers form which separate the two distinct regions of ion-ion core and ion-electron priority [1-8].

Adding the electronegative ions into the discharge, the plasma reveals the new features which depend on the electronegativity condition. Investigations in recent decades on electronegative plasma have shown for some ranges of the electronegativity, the Bohm's criterion becomes 
multivalued, and before the sheath formation, the profiles of the plasma parameters such as density and electric potential become oscillatory $[9,10]$.

In addition, in many situations such as magnetic confinement fusion and material processing, the impact of the magnetic field on the plasma-wall interaction should be considered. The magnetic field leads to a change in the structure of the sheath and presheath. For example, in Refs. [11-13], it is shown that the magnetization leads to a decrease in the positive ion flux and saturation current collected by negative electrode and positive ions deviates from the perpendicular to the wall.

It is to be noted that, in many cases of interest such as plasma fusion devices, plasma spray, and plasma-assisted chemical vapor deposition, the temperature of positive ions in the vicinity of the walls should be considered and it should be included in the governing equations. References [14-16] consider a transition layer by adding the ion temperature and obtain the space charge characteristics in the sheath-presheath region.

The present paper is an extension of an earlier article [16], in which the influence of the adiabatic thermal flow of positive ions instead of the isothermal flow of them is studied in magnetized electronegative plasma in two special cases of collision cross sections. One of the advantages of the presence study with respect to our previous paper [16] is that it takes into account the general cross section for the neutral-positive ions collision. It will be shown that the governing model of the thermal flow of the positive ion changes the general features of the plasma sheath structure such as parameter space region and space charge profiles. The space parameter region shows the ranges of concentration and temperature of negative ions in which the plasma potential at the sheath edge becomes multivalued.

To generalize the influence of the collisions on the magnetized electronegative presheath, we have assumed that the collision cross section depends on the velocity and transition region has been treated in two special cases of collision cross section. In Sect. 2, we present the model equations using the set of hydrodynamic equations and proper assumptions. In Sect. 3, we solve the equations for different parameters and compare the results with conclusions of the cold and isothermal plasmas. In Sect. 4, we conclude the paper with a brief summary of the results.

\section{Model equation}

The dynamics of the positive ions in the electronegative plasma sheath can be described by the continuity and momentum transfer equations.

$\nabla \cdot\left(n_{i} \vec{V}_{i}\right)=Z n_{e}$,

$m_{i} n_{i}\left(V_{i} \cdot \nabla \vec{V}_{i}\right)+m_{i} n_{i} v_{c} \vec{V}_{i}=e n_{i}\left(\vec{E}+\bar{V}_{i} \times \vec{B}\right)-m_{i} Z n_{e} \vec{V}_{i}-\nabla P_{i}$.
Here, $B$ is the intensity of the applied magnetic field, $E$ is the electric field, $e$ is the elementary charge, $m_{i}$ and $V_{i}$ are the positive ions mass and velocity, respectively, $P_{i}$ is the positive ions pressure, $n_{e}$ and $n_{i}$ are the electron and negative ion densities in the sheath region, respectively, $v_{c}$ is the elastic collision frequency, and $Z$ is the ionization frequency.

The negative ions and electrons are described by the Boltzmann distributions of their own temperatures [14].

$n_{e}=n_{e 0} \exp \left(\frac{e \varphi}{k T_{e}}\right)$,

$n_{-}=n_{-0} \exp \left(\frac{e \varphi}{k T_{-}}\right)$,

where $n_{-}$is the negative ion density in the sheath, $k$ is the Boltzmann's constant, $T_{e}$ and $T_{-}$are the electron and negative ion temperature, respectively, $n_{e 0}$ and $n_{-0}$ are the electron and negative ion density in the quasi-neutral plasma, respectively, and $\varphi$ is the electric potential. The magnetic field $\vec{B}$ is embedded in $x-y$ plane as $\vec{B}=B_{0} \cos (\theta) \hat{i}+B_{0} \sin (\theta) \hat{j}$, where $B_{0}$ is the magnitude of applied magnetic field and $\theta$ is the angle between magnetic field and $x$-axis. Here, it is assumed that the $x$-axis is the normal to the wall coordinate.

The electric potential is determined self-consistently and is given by the Poisson's equation.

$\nabla^{2} \varphi=-\frac{e}{\varepsilon_{0}}\left(n_{i}-n_{e}-n_{-}\right)$,

where $\varepsilon_{0}$ is the vacuum permittivity constant. Here, we have considered a 1D sheath from sheath edge to wall. Also, it is assumed that the collision frequency depends on the positive ion velocity as

$v_{c}=n_{n} \sigma V_{i}$,

where $n_{n}$ is the neutral gas density and $\sigma$ is the collision cross section that has a power law dependence on the ion velocity of the form.

$\sigma=\sigma_{s}\left(\frac{V_{i}}{c_{s}}\right)^{p}$

For an isotropic medium, the characteristics features of the medium are same in all directions and therefore the collision cross section depends on the magnitude of the velocity (speed). However, in the presence of the magnetic field, the properties of the plasma along the applied magnetic field are different from the perpendicular direction and the plasma medium behaves such as an anisotropic medium. Therefore, we assume that the cross section is a function of the positive ion velocity [17]. The quantity $p$ is the dimensionless parameter ranging from -1 to 0. $c_{s}$ is the ion acoustic velocity and $\sigma_{s}$ is the collisional 
cross section measured at ion acoustic velocity. $p=-1$ and $p=0$ correspond to the constant collision frequency and constant mean free path.

We assume that the thermal ion flow is adiabatic. This is a convenient assumption for the present study where we treat the positive ions dynamics by the fluid model in a planar sheath [18]. The positive ion partial pressure is related to its density as $P=C n_{i}^{\kappa}$, where $\kappa=3,2, \frac{5}{3}$ for unidimensional, bidimensional, or tridimensional adiabatic flow, respectively [15]. Therefore, the partial pressure for the positive ion is related to its density as $P=k T_{i} \frac{n_{i}^{3}}{n_{i 0}^{2}}$.

We normalize the variables using the following dimensionless quantities:

$$
\begin{aligned}
\eta & =-\frac{e \phi}{k T_{e}}, \quad u_{x}=\frac{V_{x}}{c_{s}}, \quad u_{y}=\frac{V_{y}}{c_{s}}, \quad u_{z}=\frac{V_{z}}{c_{s}}, \\
\xi & =\frac{x}{s}, \quad s=\frac{c_{s}}{Z}, \quad \beta=\frac{e B}{m_{i} Z}, \quad N_{i}=\frac{n_{i}}{n_{e 0}}, \\
N_{e} & =\frac{n_{e}}{n_{e 0}}, \quad N_{-}=\frac{n_{-}}{n_{e 0}}, \quad \alpha=\frac{n_{-0}}{n_{e 0}}, \quad T=\frac{T_{i}}{T_{e}} \\
\gamma & =\frac{T_{e}}{T_{-}}, \quad q=\frac{\lambda_{D e} Z}{c_{s}}, \quad c_{s}=\sqrt{\frac{k T_{e}}{m_{i}}}, \quad \delta=\frac{n_{n} \sigma_{s} c_{s}}{Z}, \\
\varepsilon & =\frac{\partial \eta}{\partial \xi}, \quad \lambda_{D e}=\sqrt{\frac{\varepsilon_{0} k T_{e}}{n_{e 0} e^{2}}} .
\end{aligned}
$$

In a steady-state condition, the plasma adjacent to the wall can be separated into two regions: semi-quasi-neutral presheath and non-neutral positive space charge sheath. The presheath adopts the length scale according to the presheath mechanism or equivalently the dimension of the vessel confining the plasma while for the sheath region; its length is scaled to the electron Debye length. Here, as we are going to investigate the presheath region in which the ionization is the main mechanism, we scale our equations to the ionization length, based on the Riemann's work [19].

Hence, the governing equations for collisional adiabatic positive ion flux in dimensionless form can be written as:

$\left(N_{i} u_{x}\right)^{\prime}=e^{-\eta}$,

$\left(N_{i} u_{x}^{2}\right)^{\prime}=N_{i} \varepsilon-N_{i} \beta u_{z} \sin (\theta)-N_{i} \delta u_{x}^{p+2}-3 T \frac{N_{i}^{2}}{(1+\alpha)^{2}} N_{i}^{\prime}$,

$\left(N_{i} u_{x} u_{y}\right)^{\prime}=N_{i} \beta u_{z} \cos (\theta)-N_{i} \delta u_{y}^{p+2}$,

$\left(N_{i} u_{x} u_{z}\right)^{\prime}=N_{i} \beta u_{x} \sin (\theta)-N_{i} \beta u_{y} \cos (\theta)-N_{i} \delta u_{z}^{p+2}$, $\eta^{\prime \prime}=\frac{1}{q^{2}}\left(N-e^{-\eta}-\alpha e^{-\gamma \eta}\right)$.

To solve the equations via the initial value problem (IVP), the above set of equations should be transformed into a system of first-order differential equations as follows:

$N_{i}^{\prime}=\frac{e^{-\eta}}{u_{x}}-\frac{N_{i}}{u_{x}} u_{x}^{\prime}$,

$u_{x}^{\prime}=\frac{1}{N_{i}} \frac{u_{x}}{u_{x}^{2}-\frac{3 T N^{2}}{(1+\alpha)^{2}}}\left[N_{i} \varepsilon-u_{x} e^{-\eta}-N_{i} u_{z} \beta \sin (\theta)\right.$

$$
\left.-N_{i} \delta u_{x}^{p+2}-\frac{3 T N_{i}^{2}}{(1+\alpha)^{2} u_{x}} e^{-\eta}\right] \text {, }
$$

$\frac{\mathrm{d} u_{y}}{\mathrm{~d} \xi}=\frac{1}{N_{i} u_{x}}\left[N_{i} u_{z} \beta \cos (\theta)-u_{y} e^{-\eta}-N_{i} \delta u_{y}^{p+2}\right]$,

$\frac{\mathrm{d} u_{z}}{\mathrm{~d} \xi}=\frac{1}{n u_{x}}\left[N_{i} u_{x} \beta \sin (\theta)-N_{i} u_{y} \beta \cos (\theta)-u_{z} e^{-\eta}-N_{i} \delta u_{z}\right]$,

$\frac{\mathrm{d}^{2} \eta}{\mathrm{d} \xi^{2}}=\frac{1}{q^{2}}\left(N_{i}-N_{e}-N_{-}\right)$.

\section{Results and discussion}

As mentioned in "Introduction" section, in the electronegative plasmas, there is a range that the plasma presheath has an oscillatory behavior and the velocity and the Bohm's criterion becomes multivalued. Therefore, we have to solve the governing equations via the initial value problem instead of boundary value problem (BVP). Therefore, we have to specify the initial values of unknown variables of the governing equations. We summarize the set of initial values of density, velocity, and potential as $\eta=0, \frac{\mathrm{d} \eta}{\mathrm{d} \xi}=0$, and $u_{y}=u_{z}=0$, respectively, at the sheath edge for a constant magnetic field angle of $\theta=\pi / 3$.

In semi-quasi-neutral presheath, the density of positive ions is slightly greater than negative species. From the set of Eqs. 13-17, it can be shown that the initial value of the positive ion density at the sheath edge is

$2 q^{2}+n_{0} \delta q^{2}+n_{0}^{2} q^{2} \beta^{2} \sin ^{2} \theta=\left(n_{0}\right)^{2}\left(n_{0}-1-\alpha\right)$.

For small values of non-neutrality $q$, one can see that $n_{0}=1+\alpha$.

It is to be noted that, for cold ions and in the absence of the ionization collisions, an analytical solution can be found 
with relatively small difficulties. However, in the presence of thermal positive ions and ionization collisions, no continuous solution can be found in the plasma sheath [20].

However, as can be seen from Eqs. 13 and 14, the initial value for the positive ion velocity in the sheath direction should satisfy the inequality of $u_{x}^{2}>\frac{3 T N^{2}}{(1+\alpha)^{2}}$ to avoid the singularities in the equations.

In comparison with isothermal presheath [16], it can be seen that for adiabatic thermal flow, the positive ions have the greater velocity to enter the non-neutral presheath.

Here, we are going to investigate the influence of the external factors such as applied magnetic field on the plasma parameters such as density, velocity, and electric potential. Figure 1 shows the density of the cold positive ions, electrons, and negative species $\left(N_{i}, N_{e}\right.$, and $N_{-}$, respectively), in the absence of the collisions for an intermediate magnetic field $\beta=1.5, \gamma=30$, and $\alpha=1.5$.

This figure shows the usual feature of the plasma sheath in which the surrounding wall repels the negative species and near the wall, there would be a positive space charge region. Furthermore, the negative ions which have the lower temperature with respect to the electrons cannot follow the electrons and therefore, near the plasma wall, the sheath is electropositive.

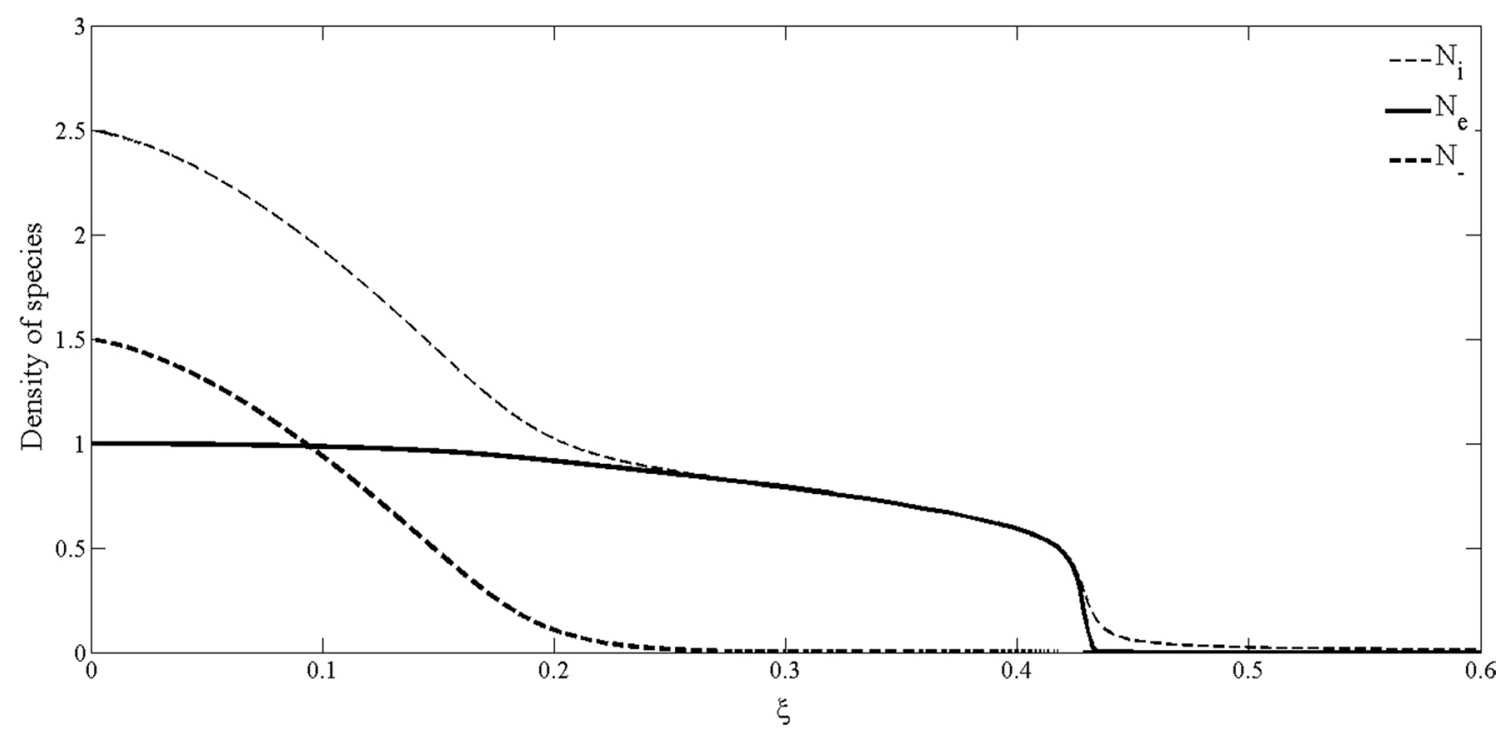

Fig. 1 The profile of the species density for collisionless cold positive ions for $\beta=1.5, \gamma=30, \alpha=1.5$

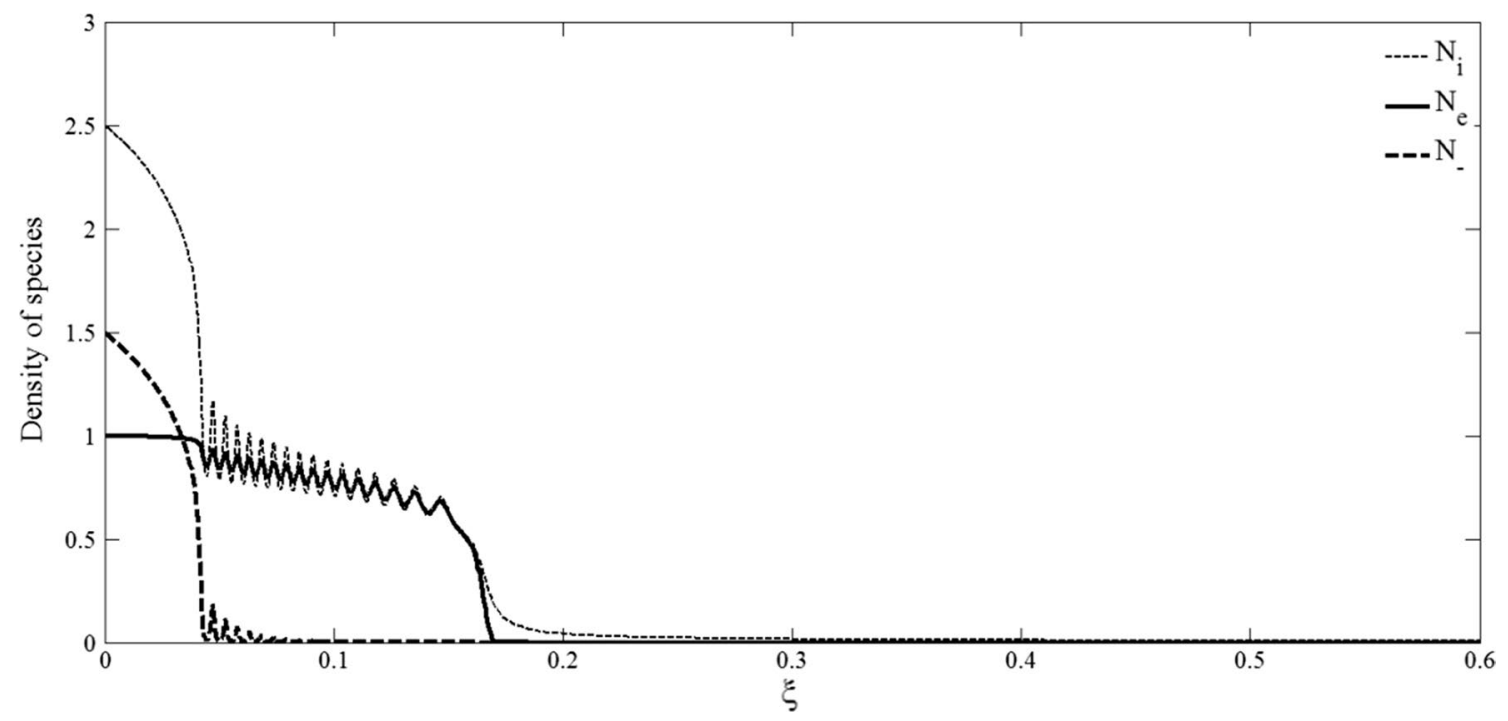

Fig. 2 The profile of the species density for collisionless warm positive ions $(T=0.005)$ for $\beta=1.5, \gamma=30, \alpha=1.5$ 
Keeping the same parameters, by an increase in the positive ion temperature and for $T=0.005$, the influence of the temperature on the density profiles is investigated. As shown in Fig. 2, some oscillations appear in the positive ion density. These oscillations are the main feature of the formation of the multilayers and are the consequences of multivaluation of the electric potential at the plasma sheath edge. In addition, increasing the positive ion temperature leads to contraction of plasma sheath thickness. It is to be noted that in the positive space charge sheath region where the ion flux density is an invariant quantity and there are no ionization collisions, increasing the ion temperature leads to increasing the sheath thickness [21]. However, in the sheath region where the ionization collisions take place (presheath region), the sheath thickness is a decreasing function of the positive ion temperature $[2,16]$.

By further increase in the positive ion temperature, the oscillations disappear and the transition region leaves the multilayer regime as shown in Fig. 3. The same results were obtained in Ref. [16] where the positive ions are in isothermal equilibrium. But it can be concluded that for adiabatic thermal flow, the oscillatory behavior takes place in lower values of the positive ion temperature.

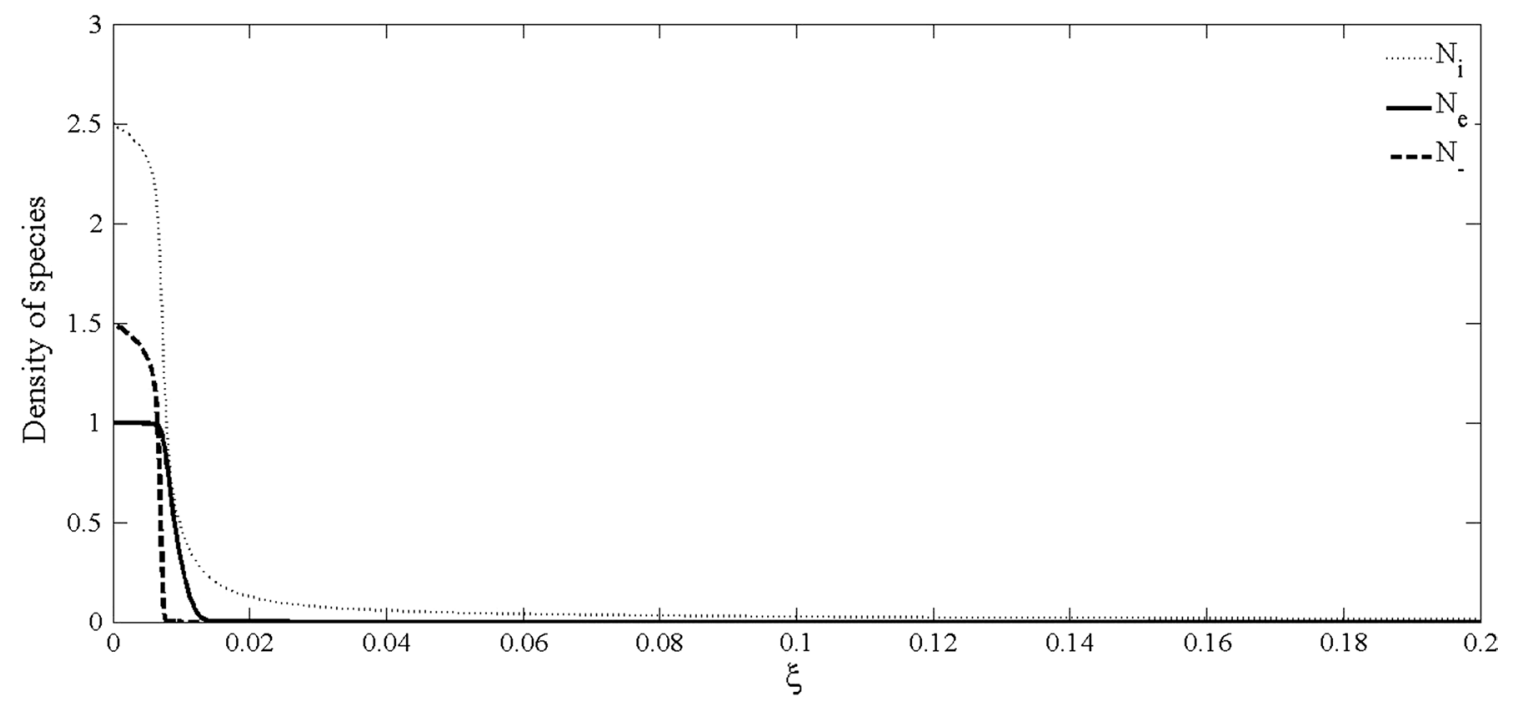

Fig. 3 The profile of the species density for collisionless hot positive ions $(T=0.02)$ for $\beta=1.5, \gamma=30, \alpha=1.5$

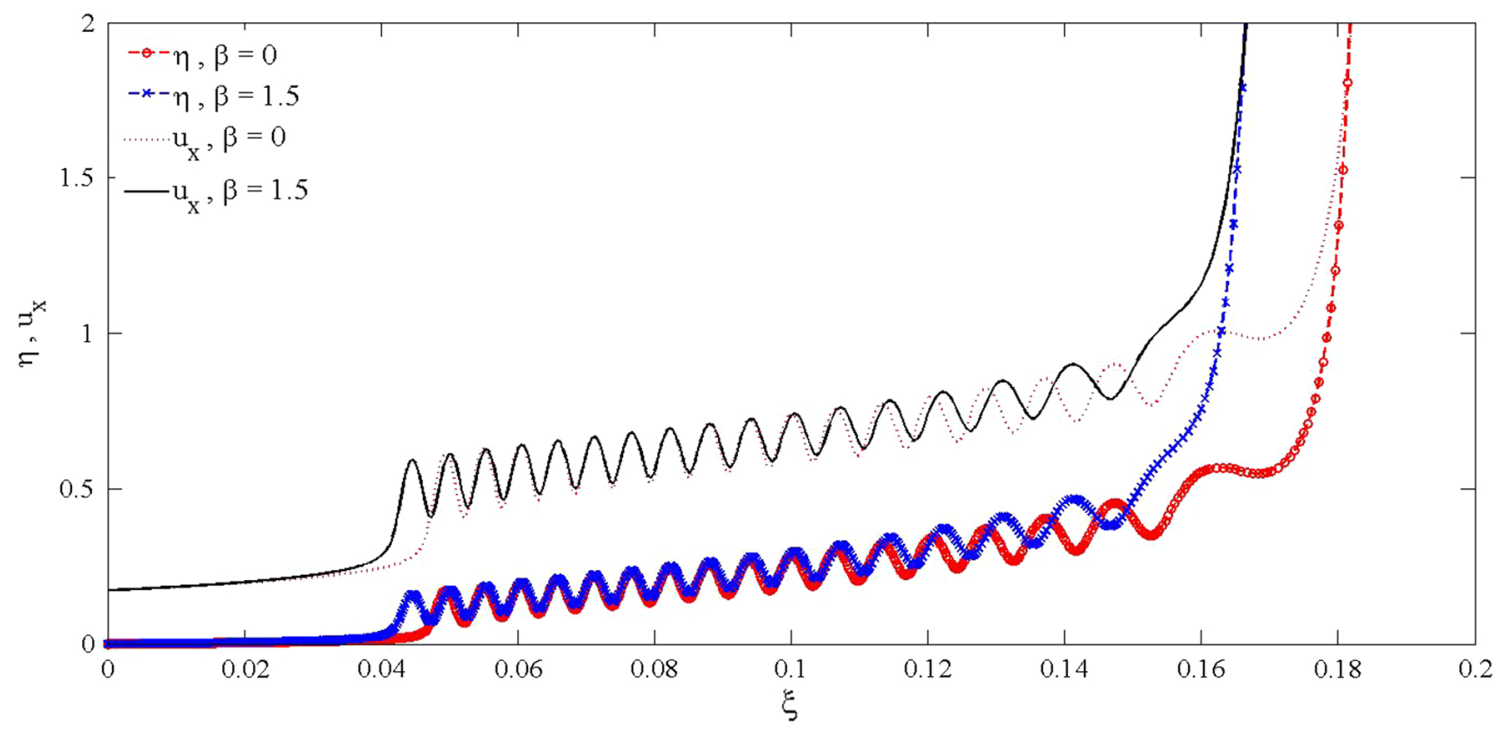

Fig. 4 The profile of the positive ion velocity and electric potential for collisionless warm positive ions $(T=0.005, \gamma=30)$ for magnetized and non-magnetized discharges 
To investigate the influence of the magnetic field on the stratified multiple transition layer, the profiles of the electric potential and positive ion velocity are obtained for two values of magnetic field when $T=0.005$. As shown in Fig. 4 , in both profiles, there are some oscillations which are the results of the formation of multilayers. The magnetic field leads to decreasing the sheath thickness, but has no influence on the oscillations wavelength. However, in the presence of the magnetic field, for positive ions attains the required velocity (Bohm's criterion) in shorter distance to enter the non-neutral positive space charge sheath.

One of the consequences of applying the magnetic field on the plasma is the formation of the drift velocity perpendicular to sheath axis which causes flux in $E \times B$ direction. Therefore, it is useful to obtain the positive ion velocity parallel $\left(V_{||}\right)$and perpendicular $\left(V_{n}\right)$ to applied magnetic field direction. In Fig. 5, the profiles of density and also the parallel and perpendicular velocity are shown for

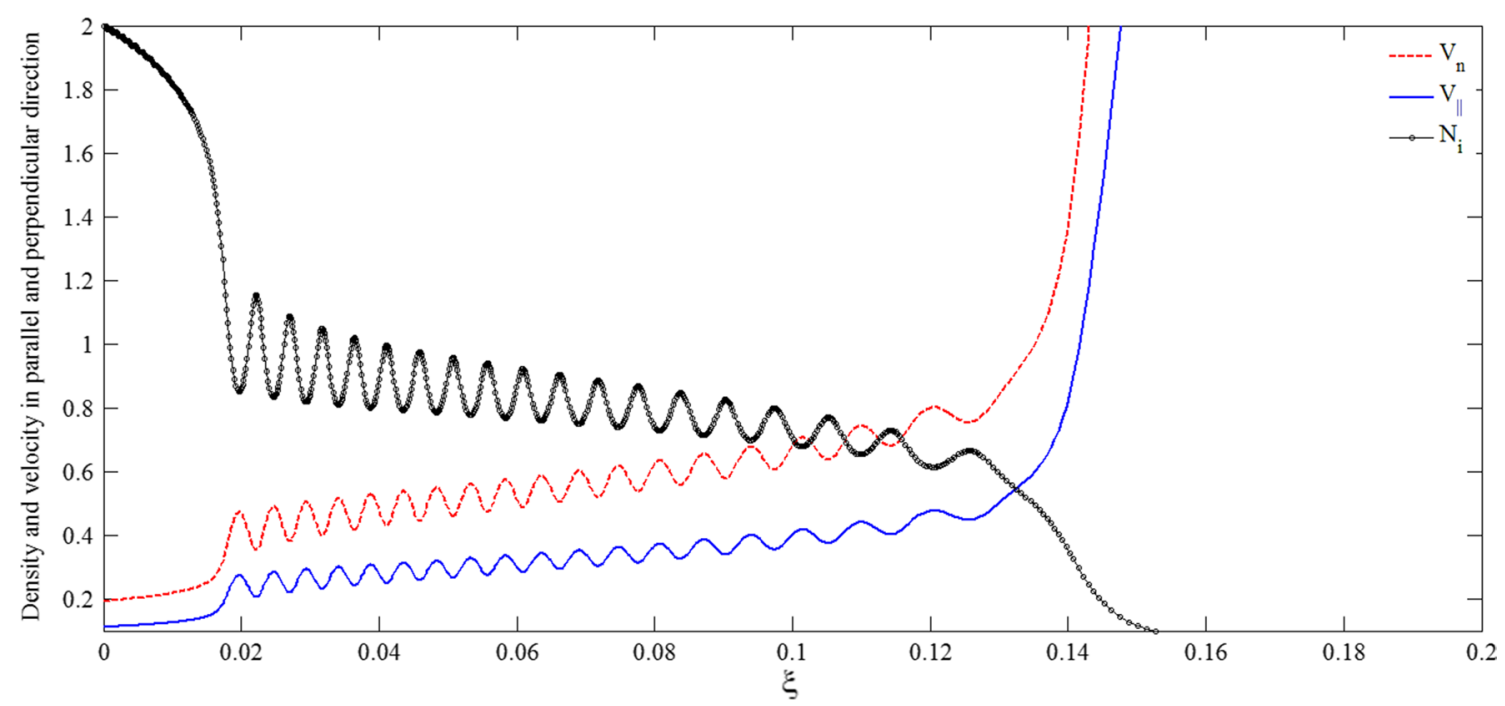

Fig. 5 The profile of density and parallel and perpendicular positive ion velocity for hot positive ions $(\beta=3, \alpha=1, T=0.01, \gamma=35)$ in collisionless condition

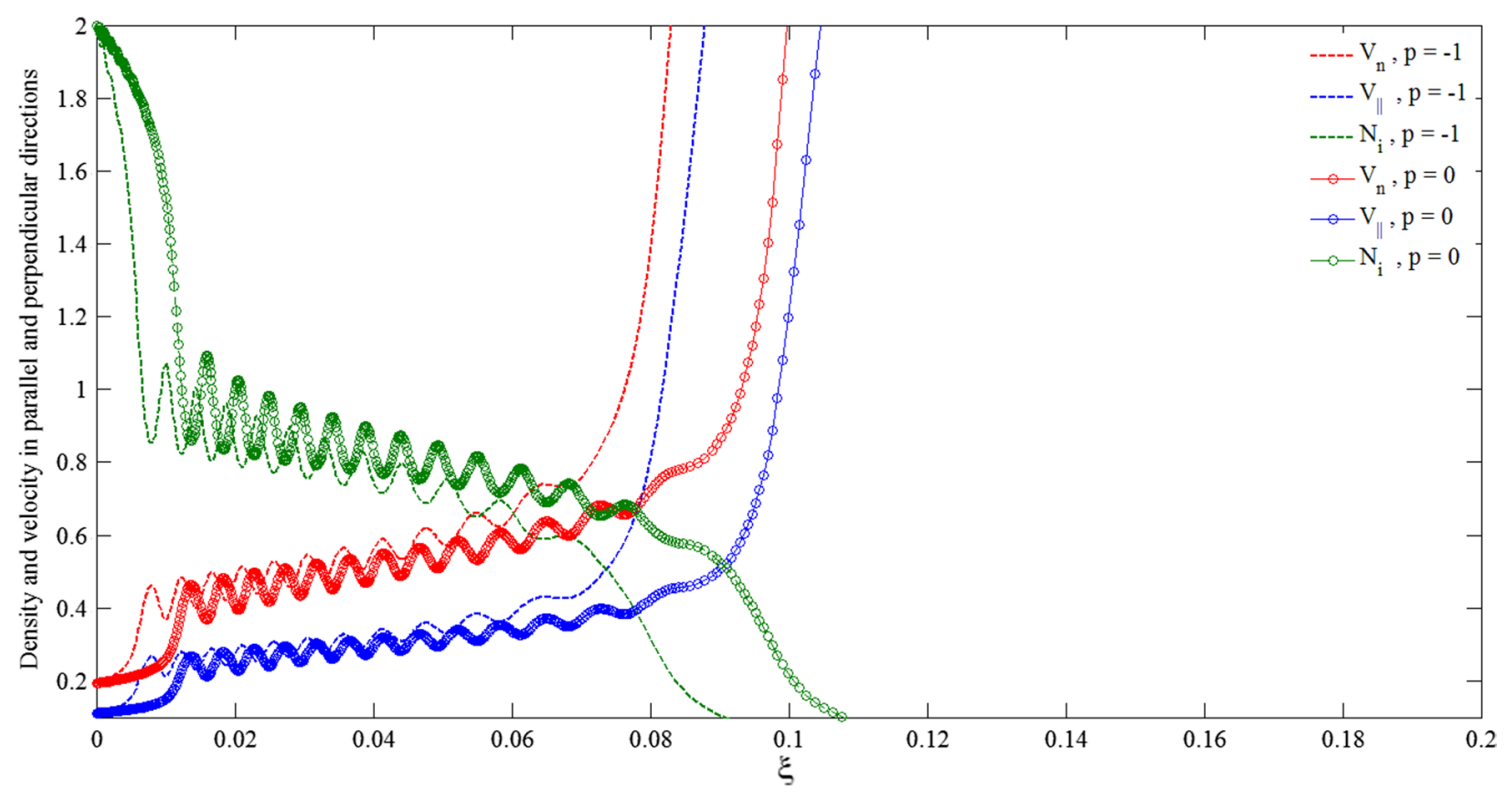

Fig. 6 The profile of density and parallel and perpendicular positive ion velocity for warm positive ions for $\beta=3, \alpha=1, \gamma=35, \delta=4, T=0.01$, and $p=0,-1$ 
$\beta=3, \alpha=1, \gamma=35$, and $T=0.01$ in a collisionless condition. In the middle of the plasma presheath region, the components of the parallel and perpendicular of velocity diverge from each other. But the oscillatory behavior takes place in two directions.

However, the collisions change the values of the drift velocity across the sheath axis. In Fig. 6, the profiles of the density and parallel and perpendicular positive ion velocity are presented for $\beta=3, \alpha=1, \gamma=35$ when $\delta=4$ for constant collision frequency and constant collision cross section.

By comparison of Fig. 5 with Fig. 6, it can be concluded that the presence of the collision leads to the attenuation of the $E \times B$ drift flow and collisions attenuate the influence of the magnetic field. In addition, by comparison of constant collision cross section with constant collision frequency, it can be seen that when the collision frequency is constant, the influence of the collisions is more pronounced.

As we are investigating the presheath region and we have scaled the sheath region to the ionization length, the sheath edge is located where the electric potential becomes infinite. Therefore, one can conclude that when $p=-1$, the presheath length decreases and positive ions can reach the plasma wall in lower distance which is the consequence of the formation of higher self-consistent electric potential. According to Ref. [11], the external magnetic field and collisions might have similar influences on the discharge structure. By variation of the magnetic field, the structure of the discharge change and switch from the uniform to multilayers for moderate magnetic field strength and for stronger magnetic field discharge might leave this regime. These are the results of the deceleration of positive ion along the sheath axis.

In addition, as mentioned before, the collisions also cause the contraction of the sheath. Furthermore, the influence of the collisions far from the sheath edge is more pronounced with respect to the near the edge.

To focus on the influence of the positive ion temperature on the collisional magnetized sheath, the density and velocity of the positive ions are shown in Figs. 7 and 8 for two special cases of constant collision cross section and constant collision frequency. From these figures, it can be seen that the density and velocity have the mirror symmetry approximately and by an increase in the temperature the sheath thickness and number of oscillations decrease. Moreover, the influence of the collisions on the positive ion acceleration is more pronounced for constant collision frequency.

In the presheath region, the flux of the positive ions increases and reaches a constant value at the beginning of the sheath edge where in the sheath region, due to the lack of the ionization, the flux is invariant. But, in the presence of the magnetic field, the positive ions deflect from the presheath-sheath axis. In Fig. 9, the influence of the negative ion, positive ion temperature on the flux in sheath axis $\left(\Gamma_{x}\right)$, and also parallel to the magnetic field $\left(\Gamma_{\|}\right)$is shown. By adding the negative ions, the positive ion flux increases which is in agreement with the results of Ref. [11]. It is interesting that in cold plasma, the flux along the $\mathrm{x}$-axis increases and in the middle of the presheath attenuates. In addition, the temperature of the positive ion leads to an increase in the parallel flux.

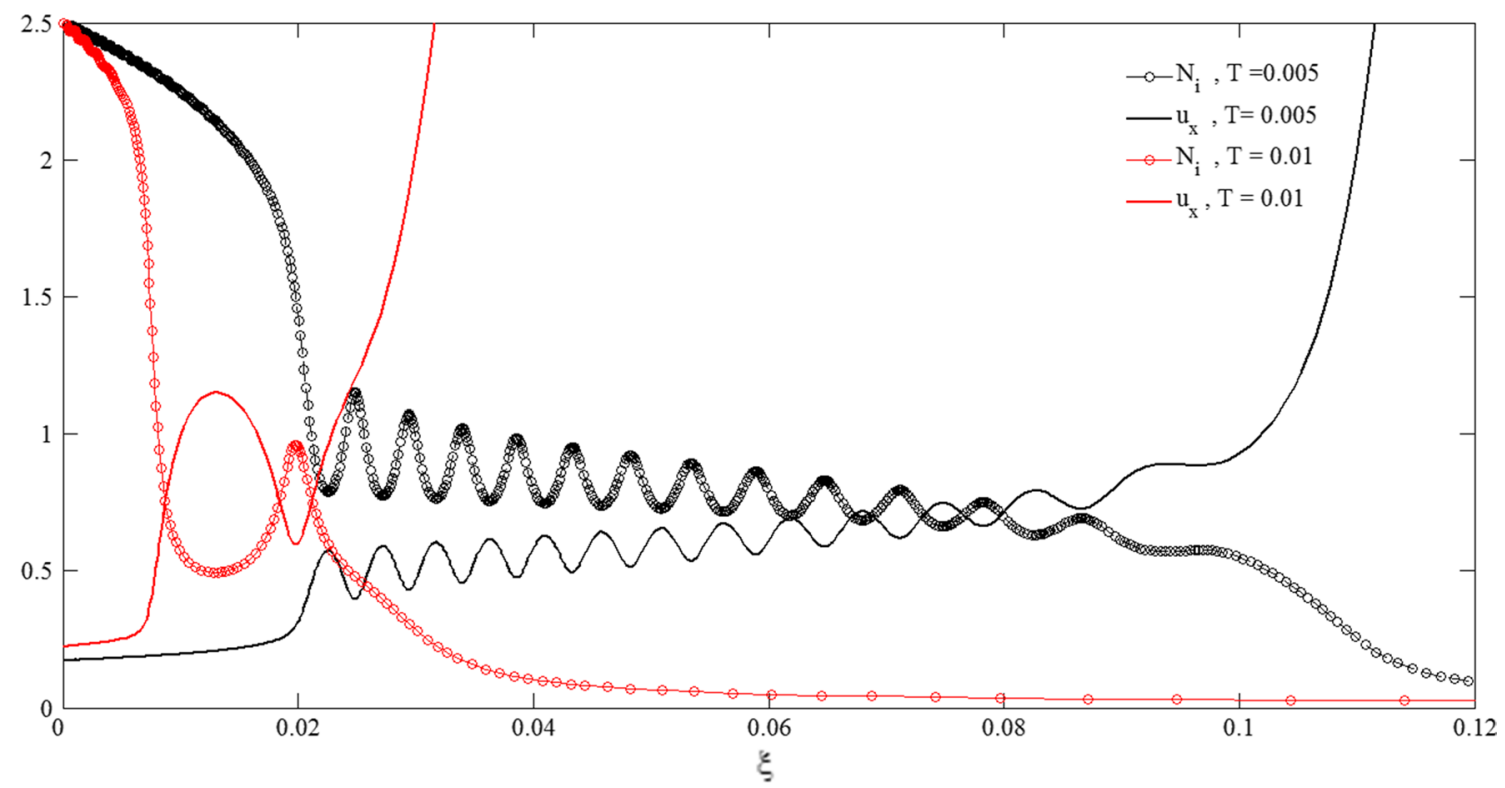

Fig. 7 The profile of the positive ion velocity and density for $\beta=1.5, \gamma=35, \alpha=1.5, p=0$ for two values of $T=0.01$ and $T=0.005$ 


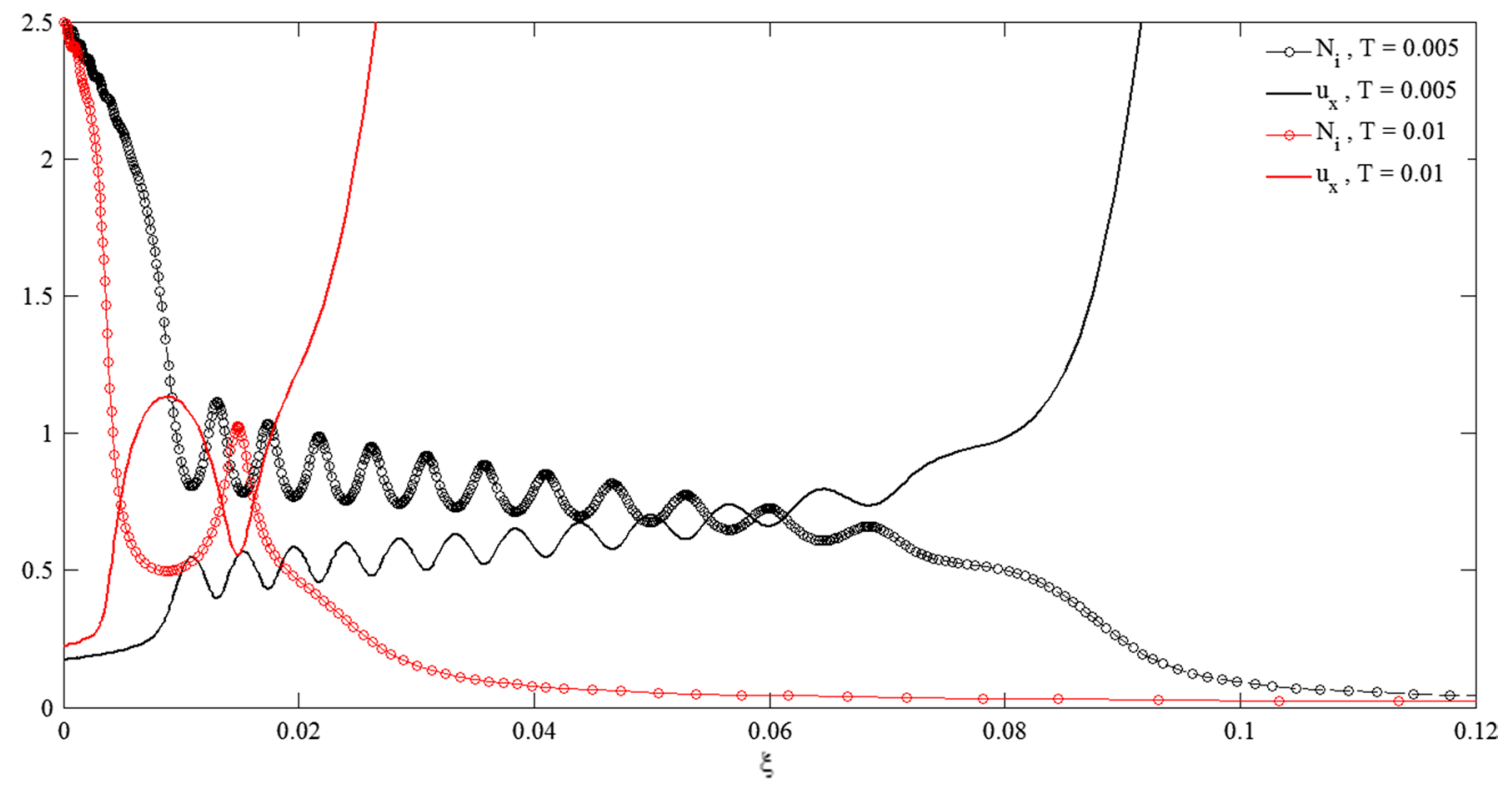

Fig. 8 The profile of the positive ion velocity and density for $\beta=1.5, \gamma=35, \alpha=1.5, p=-1$ for two values of $T=0.01$ and $T=0.005$

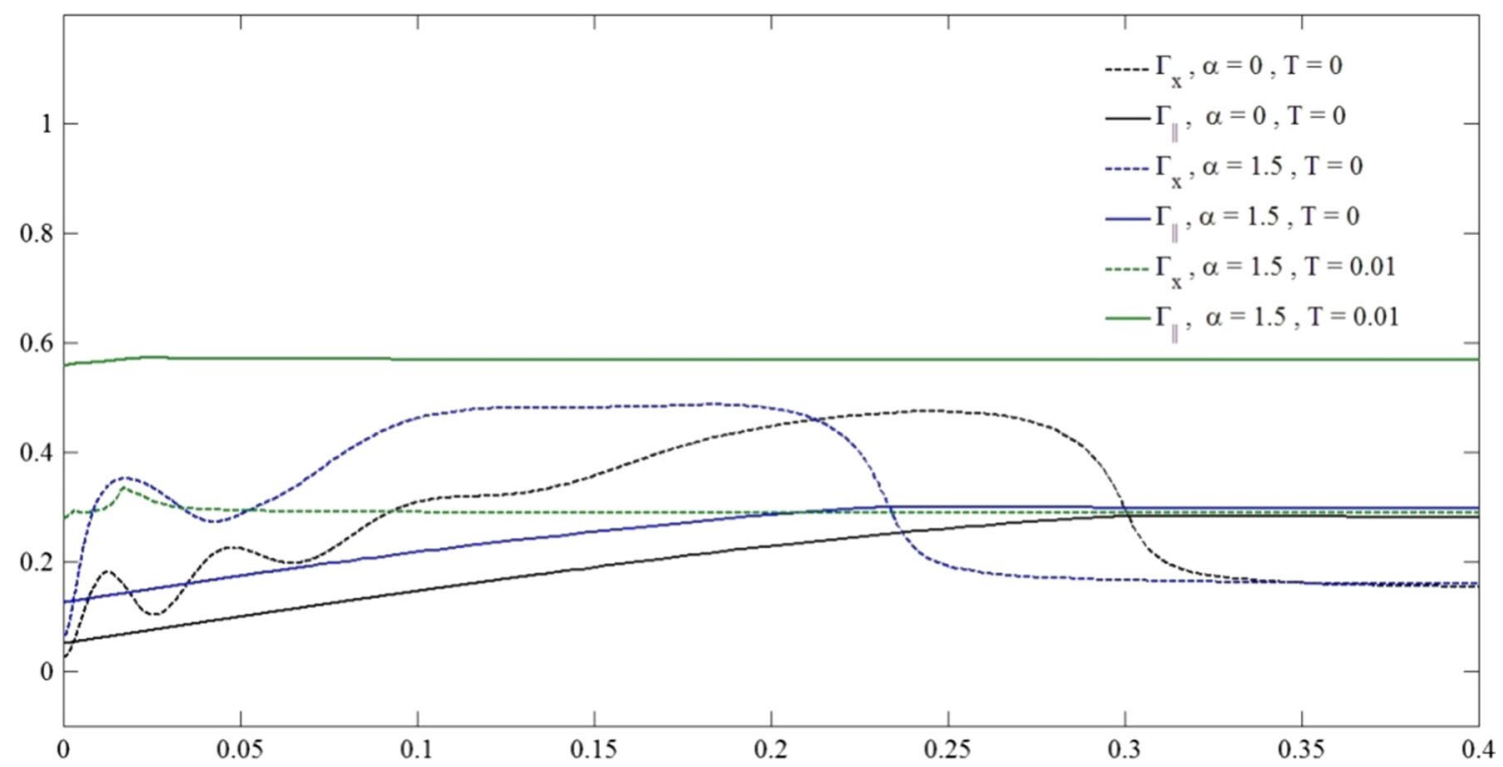

Fig. 9 The profile of flux profile in collisionless case when $\gamma=35$ in parallel and perpendicular directions

As mentioned in "Introduction," the space charge region influences the energy and the dose of the accelerated positive ions through sheath. The formation of the space charge has a great influence on the result of Langmuir probe and the extracted ion beam from the plasma ion source. According to Fig. 10, adding the negative ions and applying the magnetic field lead to moving the space charge peak to the sheath edge. However, the presence of the thermal positive ions leads to appearance of some oscillations related to the formation of multilayer structure. The collisions and the model of the collision cross section have no influence on the oscillation, and discharge cannot leave multilayer structure. In addition, it can be seen that the temperature of the positive ions is responsible for oscillations and the magnetic field has no influence on the appearance of such behavior.

However, it is important to find the plasma parameters in which the transition region becomes multilayer. In Figs. 11 and 12, the $\alpha-\gamma$ spaces representing the ranges of electronegativity and negative ion temperature in which sheath becomes multilayer are shown. The lower and upper 


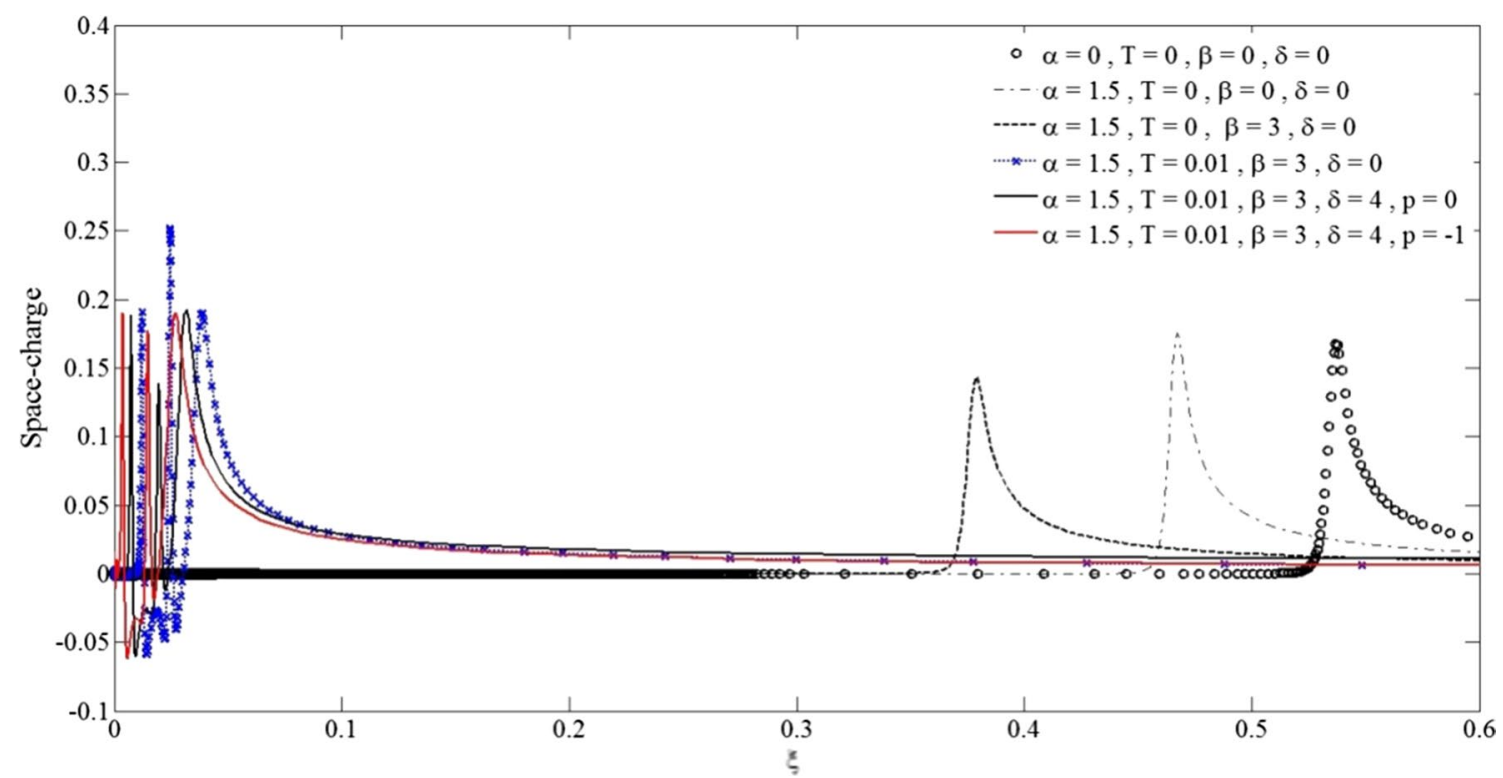

Fig. 10 The profile space charge for different plasma parameters when $\gamma=35$

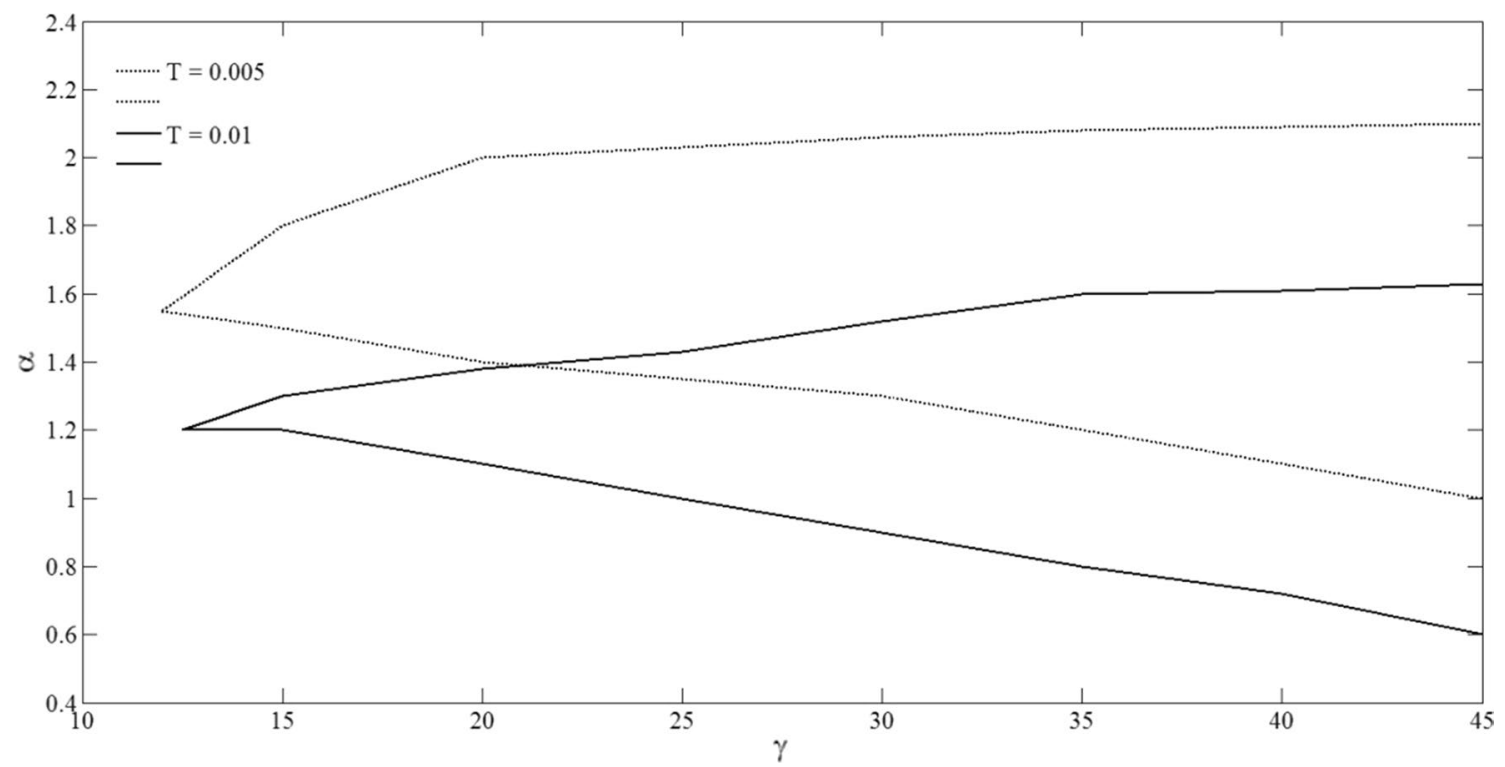

Fig. 11 The collisionless parameter space region for two values of positive ions temperature when $\beta=1.5$

branches determine the minimum and maximum values of the electronegativity in which the sheath has the oscillatory behavior. In Fig. 11, the parameter space regions are obtained for $T=0.005$ and $T=0.01$ for moderate magnetic field strength of $\beta=1.5$. As shown in Fig. 9, by the increase in the temperature, the parameter space region starts in higher values of $\gamma$ and the interior of the parameter space region occupies lower values of $\alpha$. To investigate how the magnetic field influences the parameter space region of a thermalized sheath, these spaces are sketched for low and high magnetic field strengths in Fig. 12. From Fig. 12, it can be observed that magnetic field leads to multilayer stratified structure starting at lower values of $\gamma$. In addition, the magnetic field leads to shifting the space region branches up to the higher values of electronegativity. By comparison of these results with Ref. [16], it can be deduced that for adiabatic positive ion flux, the multilayer starts for lower values of positive ion temperature 


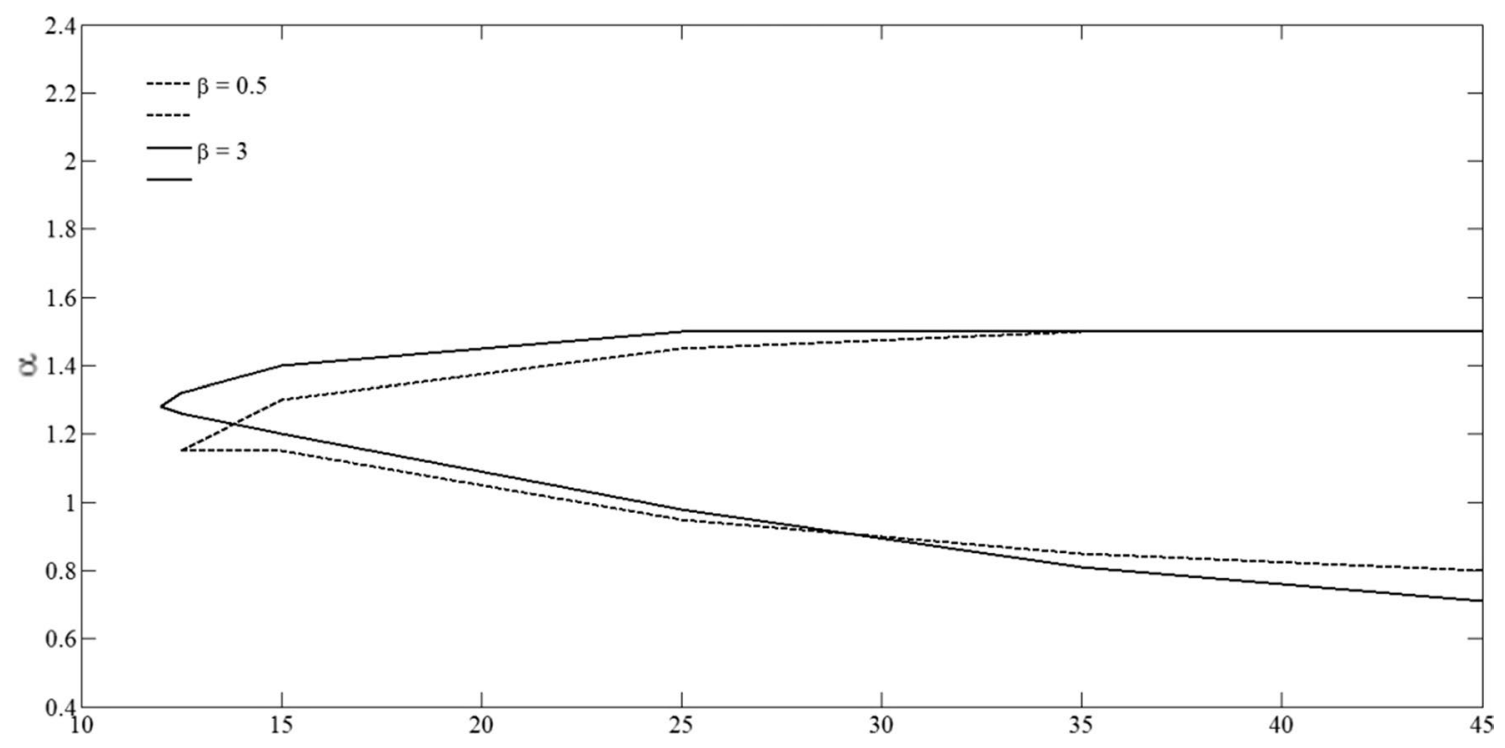

Fig. 12 The collisionless parameter space region for two values of magnetic field when $T=0.01$

and by slightly increasing the temperature, the oscillatory behaviors take place.

\section{Conclusions}

The behavior of collisional magnetized electronegative plasma ion source near the confining plasma walls is investigated in the presence of the adiabatic thermal positive ion. A power law dependency for the collision cross section is assumed, and the semi-quasi-neutral sheath is studied for constant collision frequency and constant collision cross section.

Using the fluid equations for positive ion dynamic and the Boltzmann distributions for negative species, the profiles of the density and velocity of positive ions for different values of temperature, magnetic field, and collision frequency are obtained. In addition, the $\alpha-\gamma$ space regions representing the range of the negative ion temperature and electronegativity in which the plasma sheath structure becomes multilayer stratified are obtained. The influence of magnetic field and temperature on parameter space region is investigated and compared with the isothermal positive ions.

It is shown that for adiabatic positive ion flux, the lower value of the positive ion temperature is needed for the formation of stratified multilayer structure. Because of the presence of the $E \times B$ drift, the positive ions can fulfill Bohm's criterion in smaller distance from the presheath edge with respect to the non-magnetized plasma sheath.

The influence of the positive ion temperature and collisions on acceleration and broadening of transition region is similar to the magnetic field and leads to decreasing the presheath thickness. The magnetic field does not have the influence on the amplitude of oscillations of the density and just results in contraction of those oscillations. In addition, the positive ion temperature leads to shifting the branches of parameter space region down to the lower values of electronegativity while the magnetic field leads to shifting up. The fluid model also shows that by adding the electronegative gas into the plasma ion sources and increasing the positive ion temperature, the flux of the extracted positive ion along the plasma sheath can be amplified.

Open Access This article is distributed under the terms of the Creative Commons Attribution 4.0 International License (http://creativeco mmons.org/licenses/by/4.0/), which permits unrestricted use, distribution, and reproduction in any medium, provided you give appropriate credit to the original author(s) and the source, provide a link to the Creative Commons license, and indicate if changes were made.

\section{References}

1. Sarma, B.K., Sarma, A., Bailung, H., Chutia, J.: Observation of sheath phenomena in multicomponent plasma with negative ions. Phys. Lett. A 244(1-3), 127-132 (1998)

2. Shah, S., Bandyopadhyay, M.: Effect of surface produced negative ions on near wall sheath. Plasma Phys. Control. Fusion 51, 03501 (2009)

3. Bailung, H., Boruah, D., Pal, A.R., Chutia, J.: Characteristics of presheath in multicomponent plasma with negative ions. Phys. Lett. A 333(1-2), 102-109 (2004)

4. Kawamura, E., Lieberman, M.A., Lichtenberg, A.J., Graves, D.B.: Two-dimensional simulation of inductive-capacitive transition instability in an electronegative plasma. Plasma Sources Sci. Technol. 21(4), 045014 (2012) 
5. Nozawa, T., Kinoshita, T., Nishizuka, T., Narai, A., Inoue, T., Nakaue, A.: The electron charging effects of plasma on notch profile defects. Jpn. J. Appl. Phys. 34(4S), 2107 (1995)

6. Hashimoto, K.: Charge damage caused by electron shading effect. Jpn. J. Appl. Phys. 33(10R), 6013 (1994)

7. Fujiwara, N., Ogino, S., Maruyama, T., Yoneda, M.: Charge accumulation effects on profile distortion in ECR plasma etching. Plasma Sources Sci. Technol. 5(2), 126 (1996)

8. Shindo, H., Sawa, Y., Horiike, Y.: Silicon etching employing negative ion in SF6 plasma. Jpn. J. Appl. Phys. 34(7B), L925 (1995)

9. Schott, L.: Plasma boundary layer in the presence of fast primary electrons. Phys. Fluids 30(6), 1795-1799 (1987)

10. Braithwaite, N.S.J., Allen, J.E.: Boundaries and probes in electronegative plasmas. J. Phys. D Appl. Phys. 21(12), 1733 (1988)

11. Yasserian, K., Aslaninejad, M., Ghoranneviss, M.: Structure of presheath-sheath in magnetized electronegative plasma. Phys. Plasmas 16(2), 023504 (2009)

12. Yasserian, K., Aslaninejad, M.: Parameter space region in the collisional magnetized electronegative plasma. Phys. Plasmas 17(2), 023501 (2010)

13. Crespo, R.M., Franklin, R.N.: Effect of an oblique and constant magnetic field in the sheath thickness, the floating potential and the saturation current collected by a planar wall. Plasma Sources Sci. Technol. 23(3), 035012 (2014)

14. Foroutan, G., Akhoundi, A.: Simulation study of the sheath region of a processing plasma with two-temperature electrons and charged nanoparticles. Phys. Lett. A 376(33), 2244-2251 (2012)
15. Palop, J.F., Ballesteros, J., Hernandez, M.A., Crespo, R.M., del Pino, S.B.: Sheath structure in electronegative plasmas with finite positive ion temperature. J. Appl. Phys. 95(9), 4585-4592 (2004)

16. Yasserian, K., Aslaninejad, M.: Influence of the temperature of positive ions on the sheath formation and parameter space region in magnetized electronegative plasmas. Phys. Lett. A 378(37), 2757-2762 (2014)

17. Yasserian, K., Aslaninejad, M.: Effect of the positive ion collisions on the positive space-charge in electronegative plasmas. Eur. Phys. J. D 67(8), 161 (2013)

18. Riemann, K.U.: The Bohm criterion and sheath formation. J. Phys. D Appl. Phys. 24(4), 493 (1991)

19. Reimann, K.U.: Theory of the plasma-sheath transition in an oblique magnetic field. Contrib. Plasma Phys. 34, 127-132 (1994)

20. Valentini, H.B., Kaiser, D.: The singularity of the two-fluid plasma equations, its relations to boundary conditions, and the numerical solution of these equations. Phys. Plasmas 24(12), 123508 (2017)

21. Khoramabadi, M., Ghomi, H., Ghoranneviss, M.: Effects of ion temperature on collisional DC sheath in plasma ion implantation. J. Plasma Fusion Res. Ser. 8, 1399-1402 (2009)

Publisher's Note Springer Nature remains neutral with regard to jurisdictional claims in published maps and institutional affiliations. 